\title{
BREAKDOWN OF THE DE GENNES SCALING IN $\mathrm{Y}_{1-x} \mathrm{Dy}_{x} \mathrm{Ni}_{2} \mathrm{~B}_{2} \mathrm{C}$
}

\author{
Z. Drzazga, A. Winiarska, A. Winiarski \\ Institute of Physics, University of Silesia, Uniwersytecka 4, 40-007 Katowice, Poland \\ AND D. KACZOROWSKI \\ Institute of Low Temperature and Structure Research, Polish Academy of Sciences \\ P.O. Box 937, Wrocław 2, Poland
}

\begin{abstract}
Single crystals of $\mathrm{Y}_{1-x} \mathrm{Dy}_{x} \mathrm{Ni}_{2} \mathrm{~B}_{2} \mathrm{C}(1 \leq x \leq 0.7)$ were examined by $\mathrm{X}$-ray diffraction and stoichiometry was attested by XPS measurements. The superconducting transition temperature $T_{\mathrm{c}}$ and Neel temperature $T_{\mathrm{N}}$ were determined by means of magnetisation measurements. A dramatic breakdown of the de Gennes scaling in the Dy-rich compounds was found when $i_{\mathrm{c}}<T_{\mathrm{N}}$.
\end{abstract}

PACS numbers: 74.25.Ha, 74.72.-h, 82.80.Pv

\section{Introduction}

The physical properties of $\mathrm{RNi}_{2} \mathrm{~B}_{2} \mathrm{C}$, where $\mathrm{R}=\mathrm{Y}$, Lu or rare earth, have been the subject of many recent studies [1-3]. The structure of these compounds is a body-centred tetragonal $\mathrm{ThCr}_{2} \mathrm{Si}_{2}$-type structure with additional carbon atoms in the rare earth plane. Superconductivity is observed for non-magnetic elements $\mathrm{Lu}$ and $\mathrm{Y}$ as well as for heavy magnetic rare earth. The superconducting transition temperature $T_{\mathrm{c}}$ decreases across the $\mathrm{RNi}_{2} \mathrm{~B}_{2} \mathrm{C}$ series with the de Gennes factor $d G=(g-1)^{2} J(J+1)$, where $g$ is the Lande factor and $J$ is the total angular momentum of the $\mathrm{R}^{+3}$ Hund rule ground states [2]. Good overall de Gennes scaling for the antiferromagnetic ordering Neel temperature $T_{\mathrm{N}}$ is also reported. These results reveal a coupling between the conduction electrons and the local magnetic moments of rare earth due to the long range $s-f$ interactions in the $\mathrm{RNi}_{2} \mathrm{~B}_{2} \mathrm{C}$ compounds. In the pseudoquaternary $\mathrm{R}_{x} \mathrm{R}_{1-x}^{\prime} \mathrm{Ni}_{2} \mathrm{~B}_{2} \mathrm{C}$, where $\mathrm{R}^{\prime}$ is $\mathrm{Y}, \mathrm{Lu}$ or the other rare earth, a more complicated correlation between $T_{\mathrm{c}}$ and $T_{\mathrm{N}}$ is observed. In the compounds diluted by a non-magnetic element a deviation from the de Gennes scaling depends on the rare earth $[4,5]$. Therefore, in this work the scaling behaviour of $T_{\mathrm{c}}$ and $T_{\mathrm{N}}$ for single crystals of $\mathrm{Y}_{1-x} \mathrm{Dy}_{x} \mathrm{Ni}_{2} \mathrm{~B}_{2} \mathrm{C}$ has been studied. 


\section{Experimental and results}

$\mathrm{Y}_{1-x} \mathrm{Dy}_{x} \mathrm{Ni}_{2} \mathrm{~B}_{2} \mathrm{C}$ compounds were prepared by arc melting of high purity constituents and next by prolonged annealing at $920^{\circ} \mathrm{C}$ for one week. Good quality plates like crystals with dimensions of about $1 \times 1 \times 0.1 \mathrm{~mm}^{3}$ have been extracted from the samples. The crystals were examined with X-ray diffraction. The XPS measurements were carried out with a multitechnique electron spectrometer PHI $5700 / 660$ from Physical Electronics on broken, scraped or sputtered samples. The magnetic measurements were carried out as a function of external magnetic field up to $5 \mathrm{~T}$ in the temperature range $1.7-300 \mathrm{~K}$ using a SQUID magnetometer.

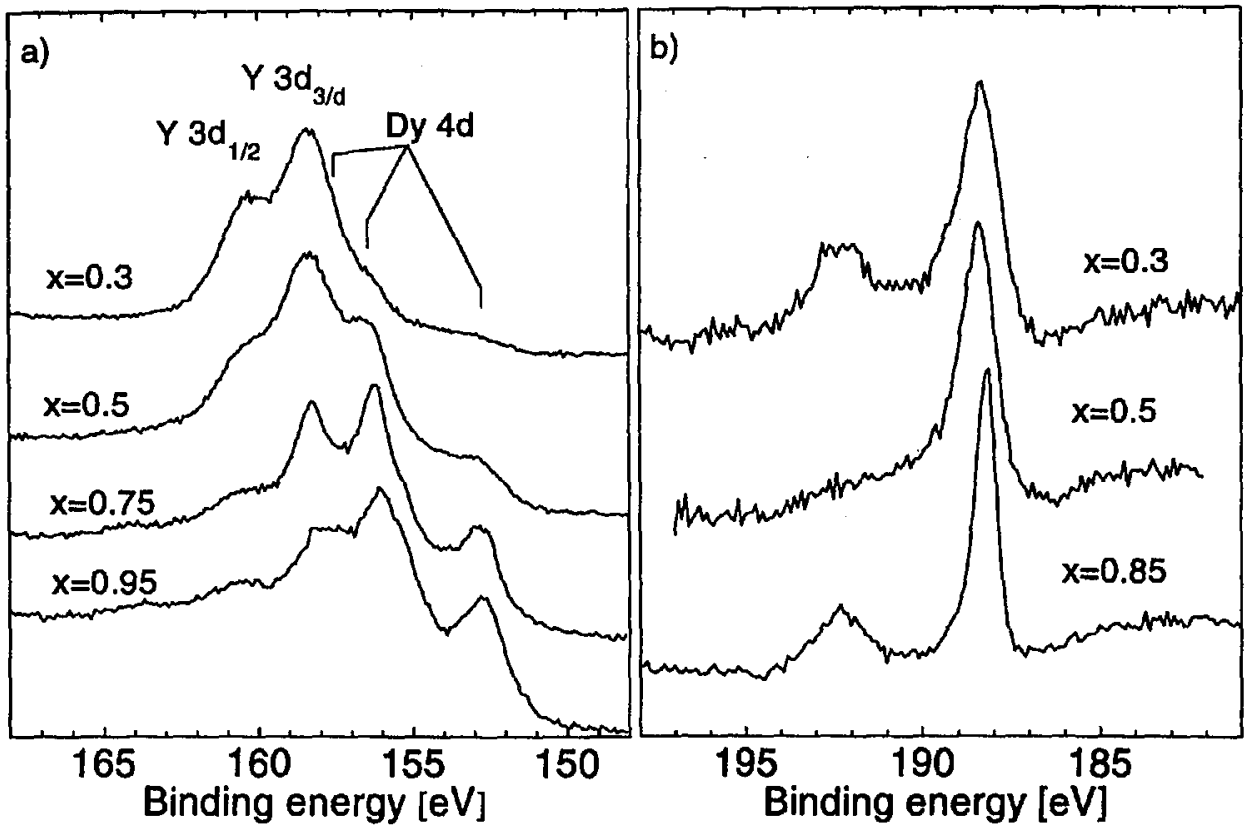

Fig. 1. (a) Overlapping the $\mathrm{Y} 3 d$ and Dy $4 d$ photoelectron spectra, (b) B $1 s$ spectra of $\mathrm{Y}_{x} \mathrm{Dy}_{1-x} \mathrm{Ni}_{2} \mathrm{~B}_{2} \mathrm{C}$.

The atomic concentrations of the investigated samples were calculated from the XPS spectra using the PHI program. Overlapped features of Y $3 d$ and Dy $4 d$ (Fig. 1a) as well as $\mathrm{Y} 3 p$ and Dy $4 p_{3 / 2}$ were separated to calculate the contribution of each feature to the measured spectra. The atomic concentrations of $\mathrm{Y}, \mathrm{Dy}, \mathrm{Ni}$, and $\mathrm{C}$ were found to be close to those which were assumed from technology. In some samples a certain fluctuation of the boron content was noticed. The B $1 s$ spectra obtained at various conditions are displayed in Fig. 1b. An additional weak broad feature at $192.3 \mathrm{eV}$ observed in some B $1 s$ spectra can be caused by sputtering the samples or indicates a deficit of $B$ in its crystallographic positions.

A temperature dependence of the magnetic susceptibility measured in external field of $H=50 \mathrm{Oe}$ and $1000 \mathrm{Oe}$ is presented in Fig. 2. At the lowest temperatures a diamagnetic signal was observed in magnetic field of $H=50 \mathrm{Oe}$ 

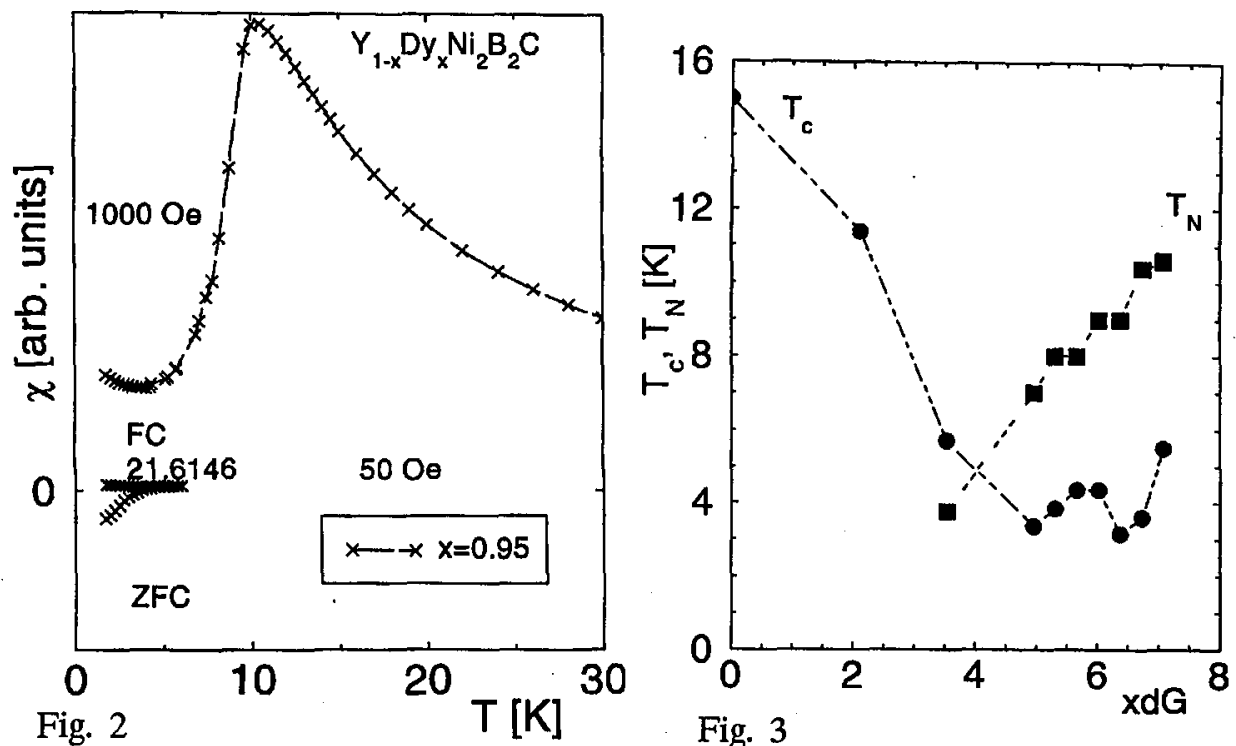

Fig. 2. Temperature dependence of dc magnetic susceptibility measured in various magnetic fields.

Fig. 3. $T_{c}$ and $T_{\mathrm{N}}$ versus $x d G\left(\mathrm{R}^{+3}\right)$ for $\mathrm{Y}_{1-x} \mathrm{D}_{x} \mathrm{Ni}_{2} \mathrm{~B}_{2} \mathrm{C}$.

for zero-field cooled samples. Superconductivity was detected for all studied concentrations but the shielding effect was weak, presumably because the applied field was of the order of the first flux penetration field [3]. Moreover, superconducting properties of these compounds are known to be sensitive to the stoichiometry of the samples [6]. Temperature in which the diamagnetic contribution vanishes in $\mathrm{ZFC}$ plots has been taken as a superconducting transition temperature. However, on cooling in the same field $(50 \mathrm{Oe})$, the $\mathrm{FC}$ plots show an anomalous behaviour, with positive values below $T_{c}$. This positive contribution in the superconducting state appears probably due to the ordering of the Dy moments above $T_{\mathrm{c}}$. Paramagnetic Meissner effect was reported earlier for ceramic $\mathrm{Bi}_{2} \mathrm{Sr}_{2} \mathrm{CaCu}_{2} \mathrm{O}_{x}$ superconductors [7]. The origin of the paramagnetic Meissner effect in the high- $T_{\mathrm{c}}$ cuprates could be spontaneous orbital current and the weak-link Josephson junctions or a pinning effect in the Kosterlitz-Thouless transition [8]. Furthermore, the dilution of the magnetic Dy sublattice by the non-magnetic $\mathrm{Y}$ atoms may enhance this unusual effect.

The temperature dependence of the dc susceptibility observed at $1 \mathrm{kOe}$ showed the characteristic peak at $T_{\mathrm{N}}$ due to antiferromagnetic ordering (Fig. 2). A comparison of the $\chi(T)$ plots proves a coexistence of superconductivity and magnetism for these compounds for $T_{\mathrm{c}}<T_{\mathrm{N}}$ as well as for $T_{\mathrm{c}}>T_{\mathrm{N}}$. Figure 3 displays $T_{\mathrm{c}}$ and $T_{\mathrm{N}}$ versus the effective de Gennes factor $x d G\left(\mathrm{R}^{+3}\right)$, where $x$ is the concentration of the magnetic ions in $\mathrm{Y}_{1-x} \mathrm{Dy}_{x} \mathrm{Ni}_{2} \mathrm{~B}_{2} \mathrm{C}$. A deviation from the linear scaling of the superconducting transition temperature $T_{\mathrm{c}}$ is observed. A breakdown of scaling is clearly seen for Dy concentration $x>0.5$ while a rather good parameterisation of magnetic pair breaking for $x<0.5$ is found. For Y-rich samples $T_{\mathrm{c}}$ 
is suppressed nearly linearly with $x d G$ according to Abrikosov-Gorkov theory. In the case of the Dy-rich samples changes in $T_{\mathrm{c}}$ are more complicated. In turn, $T_{\mathrm{N}}$ decreases gradually with an increase in nonmagnetic atoms. The non-magnetic centres could be theoretically conjectured to influence $T_{\mathrm{c}}$ due to destruction translation symmetry of antiferromagnetic dysprosium lattice [5]. On the other hand, collective magnetic excitations could be responsible for pair breaking when $T_{\mathrm{c}}<T_{\mathrm{N}}$ analogously as in $\mathrm{Ho}_{1-x} \mathrm{Dy}_{x} \mathrm{Ni}_{2} \mathrm{~B}_{2} \mathrm{C}$ [4].

A more detailed analysis of interplay between superconductivity and magnetism will be published elsewhere.

\section{Acknowledgments}

The multitechnique electron spectrometer PHI 5700/660 was partially support by the Foundation for Polish-German Cooperation, the Committee for Scientific Research and FNP-SUBIN 95 program.

\section{References}

[1] R.J. Cava, H. Takagi, H.W. Zandbergen, J.J. Krajewski, W.F. Peck Jr., T. Siegriest, B. Batlogg, R.B. van Dover, R.J. Felder, K. Mizuhasi, J.O. Lee, H. Elisaki, S. Uchida, Nature 367, 252 (1994).

[2] H. Elisaki, H. Takagi, R.J. Cava, B. Batlogg, J.J. Krajewski, W.F. Peck Jr., K. Mizuhasi, J.O. Lee, S. Uchida, Phys. Rew. B 50, 647 (1994).

[3] R. Szymczak, M .Baran, L. Gladczuk, H. Szymczak, Z. Drzazga, A. Winiarska, Physica C 254, 124 (1995).

[4] B.K. Cho, P.C. Canfield, D.C. Johnston, Phys. Rew. Lett. 77, 163 (1996).

[5] Z. Hossain, R. Nagarajan, S.K. Dhar, L.C. Gupta, Physica B 259-261, 606 (1999).

[6] C.V. Tomy, G. Balakrishan, D.McK. Paul, Physica C 248, 349 (1995).

[7] W. Braunisch, N. Knauf, V. Kataev, S. Neuhausen, A. Grutz, A. Kock, B. Roden, D. Khomski, D. Wöhlleben, Phys. Rew. Lett. 68, 1908 (1992).

[8] P. Svendlindh, K. Niskanane, P. Norling, P. Nordblad, L. Lundgren, B. Lonnberg, T. Lundstrom, Physica C 162-164, 1365 (1989). 